\title{
Automorphic compactifications and the fixed point lattice of a totally-ordered set
}

\section{Gary Davis}

\begin{abstract}
When a totally-ordered set $\Omega$ has no points fixed by all automorphisms we equip $\Omega$ with the support topology which has fixedsets of automorphisms as basic closed sets. There is a mapping from $\Omega$ into the set of prime dual ideals of the lattice $\Phi(\Omega)$ of basic closed sets and this allows us to classify the points of $\Omega$ as excellent, isolated, static, or extraordinary. There is an action of the group of automorphisms of $\Omega$ on the lattice $\Phi(\Omega)$ and this allows us to see that automorphisms of $\Omega$ preserve the prime dual ideal classification of points of $\Omega$. When the empty set is a basic closed subset of $\Omega$ the dual spectrum of $\Phi(\Omega)$ is a compact $T_{0}$ space (Hausdorff when all points of $\Omega$ are excellent) containing $\Omega$ as a dense subspace and allowing an extension of each automorphism of $\Omega$ to a homeomorphism of the dual spectrum.
\end{abstract}

\section{Introduction}

When $\Omega$ is a totally-ordered set we denote by $A(\Omega)$ the latticeordered group of order-preserving permutations of $\Omega$. If $\Omega$ is equipped with its order topology then there is always a compact Hausdorff space $X$ containing $\Omega$ as a dense subspace, having the following property:

$$
\text { every } \pi \in A(\Omega) \text { extends to a homeomorphism of } X \text {. }
$$

Received 5 November 1974. 
Namely, we can take for $X$. the lattice completion of the Dedekind completion of $\Omega$, equipped with its order topology, and then every $\pi \in A(\Omega)$ extends uniquely to an order-preserving permutation, and therefore homeomorphism, of $X$ according to

$$
\pi(x)=\bigvee\{\pi(a): a \in \Omega, a \leq x\} ;
$$

see [2].

We say that a compact $T_{0}$ space that contains $\Omega$, with some specified topology, as a dense subspace, and satisfies (*), is an automorphic compactification of $\Omega$. In [1] an automorphic compactification of $\Omega$, with its order topology, was constructed in a different way for the special case that $\Omega$ is a totally-ordered field. That construction fails in general, and it is the object of this paper to see how this situation can be repaired.

The prime dual ideal classification of points of $\Omega$

We assume throughout that $\Omega$ is a totally-ordered set. For $\pi \in A^{+}(\Omega)=\{\pi \in A(\Omega): \pi(x) \geq x$ for all $x \in \Omega\}$ we define the support of $\pi$ to be the set $\operatorname{supp}(\pi)=\{x \in \Omega: \pi(x)>x\}$, and we define fix $(\pi)=\Omega \backslash \operatorname{supp}(\pi)=\{x \in \Omega: \pi(x)=x\}$. The set $\Phi(\Omega)$ of all fix $(\pi)$, $\pi \in A^{+}(\Omega)$, is a lattice of subsets of $\Omega$, when it is ordered by inclusion, with

$$
\operatorname{fix}\left(\pi_{1}\right) \cap \operatorname{fix}\left(\pi_{2}\right)=\operatorname{fix}\left(\pi_{1} v \pi_{2}\right)
$$

and

$$
\operatorname{fix}\left(\pi_{1}\right) \cup \operatorname{fix}\left(\pi_{2}\right)=\operatorname{fix}\left(\pi_{1} \wedge \pi_{2}\right)
$$

for all $\pi_{1}, \pi_{2} \in A^{+}(\Omega)$. We call $\Phi(\Omega)$ the fixed-point lattice of $\Omega$, and denote by $K(\Omega)$ the set of prime filters of $\Phi(\Omega)$ with the hullkernel topology, which has the sets $U(\pi)=\{\xi \in K(\Omega): \operatorname{fix}(\pi) \notin \xi\}$ as basic open sets.

We observe that for each $x \in \Omega$ the set $\xi(x)=\{$ fix $(\pi): \pi(x)=x\}$ is a prime dual ideal of $\Phi(\Omega)$, and then make the following definitions: a point $x \in \Omega$ is

(1) an excellent point if $\xi(x)$ is a maximal filter, 
(2) an isolated point if $\xi(x)$ is a minimal-prime filter,

(3) an extraordinay point if $\xi(x)$ is a non-maximal and nonminimal-prime filter,

(4) a static point if $\xi(x)=\Phi(\Omega)$.

We also say that $\Omega$ is spectral if the map $x \rightarrow \xi(x)$ is injective.

LEMMA 1. The sets $\operatorname{supp}(\pi), \pi \in A^{+}(\Omega)$, are basic open sets for a topology on $\Omega$ if and only if $\Omega$ has no static points.

Proof. $\operatorname{supp}\left(\pi_{1}\right) \cap \operatorname{supp}\left(\pi_{2}\right)=\operatorname{supp}\left(\pi_{1} v \pi_{2}\right)$ for all $\pi_{1}, \pi_{2} \in A^{+}(\Omega)$, so we only need $\Omega=U\left\{\operatorname{supp}(\pi): \pi \in A^{+}(\Omega)\right\}$, and this is the case precisely when $\Omega$ contains no points $x$ satisfying $\pi(x)=x$ for all $\pi \in A^{+}(\Omega)$; that is, no static points.

When $\Omega$ has no static points we call the topology of Lemma 1 the support topology on $\Omega$.

PROPOSITION 2. If $\Omega$ has no static points then the following statements are equivalent:

(I) $\Omega$ is $T_{0}$ for the support topology;

(2) $\Omega$ is spectral;

(3) the map $x+\xi(x)$ is a homeomorphism from $\Omega$ into $K(\Omega)$.

Proof. Suppose that $\Omega$ is a $T_{0}$ space and that $\xi(x)=\xi(y)$. Then $\{\operatorname{fix}(\pi): \pi(x)=x\}=\{\operatorname{fix}(\pi): \pi(y)=y\}$ so that $x, y$ cannot be $T_{0^{-}}$ separated and therefore $x=y$. Now suppose that the map $x \rightarrow \xi(x)$ is injective. If $U(\pi)$ is a basic open set in $K(\Omega)$ then $\{x \in \Omega: \xi(x) \in U(\pi)\}=\{x \in \Omega: f i x(\pi) \notin \xi(x)\}=$

$$
=\{x \in \Omega: x \mid \operatorname{Pix}(\pi)\}=\operatorname{supp}(\pi)
$$

which is a basic open set in $\Omega$. Conversely if $\operatorname{supp}(\pi)$ is a basic open set in $\Omega$ then

$$
\{\xi(x): x \in \operatorname{supp}(\pi)\}=\{\xi(x): \operatorname{Pix}(\pi) \vDash \xi(x)\}=U(\pi) \cap\{\xi(x): x \in \Omega\},
$$

which is a basic open subset of $\{\xi(x): x \in \Omega\}$. Finally, if we assume that $x \rightarrow \xi(x)$ is a homeomorphism then $\Omega$ is a $T_{0}$ space since $K(\Omega)$ is a $T_{0}$ space; (cf. [4]). 
An important aspect of the lattice $\Phi(\Omega)$ is that the group $A(\Omega)$ acts as a group of lattice automorphisms of $\Phi(\Omega)$. Namely, we can define a homeomorphism, $\alpha$ from $A(\Omega)$ into the automorphism group of $\Phi(\Omega)$ by $\alpha(\pi) \operatorname{fix}\left(\pi^{\prime}\right)=\operatorname{fix}\left(\pi \pi^{\prime} \pi^{-1}\right)$.

PROPOSITION 3. kera is a normal sublattice subgroup of $A(\Omega)$, and as a lattice-ordered group, kera is representable.

Proof. We have to see that kera is a sublattice of $A(\Omega)$. Suppose that $\pi_{1}, \pi_{2} \in$ ker $\alpha$ and $\pi \in A^{+}(\Omega)$. If $x \in$ fix $(\pi)$ then $\pi\left(\pi_{1} \vee \pi_{2}\right)(x)=\pi_{1} \vee \pi_{2}(x)$ so that $\alpha\left(\pi_{1} \vee \pi_{2}\right)$ fix $(\pi) \subseteq$ fix $(\pi)$. We then replace $\pi_{1}, \pi_{2}$ by $\pi_{1}^{-1}, \pi_{2}^{-1}$ and see similarly that $\alpha\left(\pi_{1}^{-1} \wedge \pi_{2}^{-1}\right) \operatorname{fix}(\pi) \subseteq \operatorname{fix}(\pi)$. Then $\alpha\left(\left(\pi_{1} v_{2}\right)^{-1}\right)$ fix $(\pi) \subseteq \operatorname{fix}(\pi)$ so $\operatorname{fix}(\pi) \subseteq \alpha\left(\pi_{1} \vee \pi_{2}\right) f i x(\pi)$, and ker $\alpha$ is a sublattice. If $\pi_{1}, \pi_{2} \in A^{+}(\Omega)$ and $\pi_{1} \wedge \pi_{2}=1$ then $\operatorname{fix}\left(\pi_{1}\right) \cup \operatorname{fix}\left(\pi_{2}\right)=\Omega$, so that, for $\pi \in$ ker $\alpha$, $\operatorname{fix}\left(\pi \pi_{1} \pi^{-1}\right) \cup \operatorname{fix}\left(\pi_{2}\right)=\pi \operatorname{fix}\left(\pi_{1}\right) \cup \operatorname{fix}\left(\pi_{2}\right)=\operatorname{fix}\left(\pi_{1}\right) \cup \operatorname{fix}\left(\pi_{2}\right)=\Omega$, which gives $\pi \pi_{1} \pi^{-1} \wedge \pi_{2}=1$. In particular, with $\pi_{1}, \pi_{2} \epsilon$ kera this says that kera is representable as a lattice-ordered group.

We now see that this action of $A(\Omega)$ on $\Phi(\Omega)$ allows us to say that moving a point around by $A(\Omega)$ preserves the prime dual ideal classification of that point.

PROPOSITION 4. If $x \in \Omega$ has a given prime dual ideal classification (that is, excellent, isolated, extraordinary, static) then all points in the orbit of $A(\Omega)$ that contains $x$ have the same classification.

Proof. Since $\pi f i x\left(\pi^{\prime}\right)=\operatorname{fix}\left(\pi \pi^{\prime} \pi^{-1}\right)$ for all $\pi^{\prime} \in A^{+}(\Omega)$, $\pi \in A(\Omega)$, we see that $\xi(\pi(x))=\pi \xi(x)=\left\{\pi f i x\left(\pi^{\prime}\right): \pi^{\prime}(x)=x\right\}$. Since the elements of $A(\Omega)$ act as automorphisms of the lattice $\Phi(\Omega)$ they send maximal filters onto maximal filters, minimal-prime filters onto minimalprime filters, prime filters that are neither onto prime filters of the same type, and $\Phi(\Omega)$ onto $\Phi(\Omega)$. 
COROLLARY 5. Suppose that $A(\Omega)$ is transitive. Then all points of $\Omega$ are excellent (isolated, extraordinary) if and only if $\Omega$ has an excellent (isolated, extraordinary) point.

We say that $\Omega$ is excellent if all points of $\Omega$ are excellent.

THEOREM 6 . If $\Omega$ is $T_{0}$ for the support topology then the following conditions on $\Omega$ are equivalent:

(1) $\Omega$ is $T_{1}$;

(2) $\Omega$ is Hausdorff;

(3) for each $x \in \Omega$ there is a $\pi \in A^{+}(\Omega)$ with fix $(\pi)=\{x\}$;

(4) $\Omega$ is excellent.

Furthermore when any one of (1) - (4) holds, $\Omega$ is completely regular.

Proof. Suppose that $x, y \in \Omega$ are $T_{1}$-separated by $\operatorname{supp}(\pi)$, $\operatorname{supp}\left(\pi_{2}\right)$ respectively. Then, with $\pi_{1}^{\prime}=\pi_{1}\left(\pi_{1} \wedge \pi_{2}\right)^{-1}$ and $\pi_{2}^{\prime}=\pi_{2}\left(\pi_{1} \wedge \pi_{2}\right)^{-1}$, we have $\pi_{1}^{\prime} \wedge \pi_{2}^{\prime}=1$ and $\operatorname{supp}\left(\pi_{1}^{\prime}\right), \operatorname{supp}\left(\pi_{2}^{\prime}\right)$ Hausdorff separate $x, y$. Thus (1) and (2) are equivalent. Now suppose that $\Omega$ is Hausdorff for the support topology and $x \in \Omega$. For each $y \neq x$ there is a $\pi_{y} \in A^{+}(\Omega)$ with $y \in \operatorname{supp}\left(\pi_{y}\right)$ and $x \in \operatorname{fix}\left(\pi_{y}\right)$, so we can cover $\Omega \backslash\{x\}$ with a collection $\left\{\operatorname{supp}\left(\pi_{\lambda}\right)\right\}$ of disjoint basic open sets none of which contain $x$. Since $A(\Omega)$ is laterally complete, the join $\pi=\bigvee_{\lambda} \pi_{\lambda}$ exists and since the stabilizer subgroup $\left\{\pi^{\prime} \in A(\Omega): \pi^{\prime}(x)=x\right\}$ is closed, [3], we have $\pi(x)=x$ but $\pi(y)>y$ for $y \neq x$. Thus (2) implies (3). Now suppose that (3) holds and $\xi$ is a filter of $\Phi(\Omega)$ containing $\xi(x)$. If $\operatorname{fix}(\pi) \in \xi$ then $f i x(\pi) \cap f i x\left(\pi^{\prime}\right) \neq \square$ for all $f i x\left(\pi^{\prime}\right) \in \xi(x)$. In particular, with fix $\left(\pi^{\prime}\right)=\{x\}$, we have $\pi(x)=x$, so $\xi=\xi(x)$. Thus (3) implies (4). Finally, if $\Omega$ is excellent the map $x \rightarrow \xi(x)$ is a homeomorphism from $\Omega$ into the subspace of $K(\Omega)$ consisting of the maximal filters of $\Phi(\Omega)$, and this is a Hausdorff space. When $\Omega$ is Hausdorff an argument as in finding $\alpha \pi$ with $\pi(x)=x$ shows that $\Omega$ is completely regular.

We say that $\Omega$ is isolated if every point of $\Omega$ is isolated. 
COROLLARY 7. If $\Omega$ is isolated then $\Omega$ is not $T_{0}$ for the support topology.

\section{Functional properties of the fixed-point lattice}

The object of this section is to describe a category of totallyordered sets for which the map $\Omega \rightarrow \Phi(\Omega)$ is the object map of a contravariant functor into a category of distributive lattices.

We describe the category $A$ as follows: the objects of $\underline{\underline{A}}$ are totally ordered sets and the arrows $\phi: \Omega^{\prime}+\Omega$ are the order-preserving injections for which $\phi\left(\Omega^{\prime}\right)$ is a fixed block of $\Omega$; that is, $A(\Omega)\left(\phi\left(\Omega^{\prime}\right)\right)=\phi\left(\Omega^{\prime}\right)$.

PROPOSITION 8. The map $\Omega \rightarrow A(\Omega)$ is the object map of a contravariant functor from $\stackrel{A}{\underline{A}}$ into a category of ordered permutation groups.

Proof. Suppose that $\phi: \Omega^{\prime} \rightarrow \Omega$ is an arrow in $\underline{\underline{A}}$. Then for each $\pi \in A(\Omega)$ we can find a unique $\pi^{\prime} \in A\left(\Omega^{\prime}\right)$ making the following diagram commute:



If $\pi^{\prime}$ exists then it must be unique since $\phi \pi^{\prime}(x)=\pi \phi(x)$ for all $x \in \Omega^{\prime}$. This equation for $\pi^{\prime}(x)$ serves to define $\pi^{\prime}$ as a mapping from $\Omega^{\prime}$ to $\Omega^{\prime}$ since $\phi\left(\Omega^{\prime}\right)$ is a fixed block of $\Omega$. Then $\pi^{\prime}$ is a bifection with inverse $\left(\pi^{-1}\right)$, since

$$
\phi \pi^{\prime}\left(\left(\pi^{-1}\right)^{\prime}(x)\right)=\pi \phi\left(\left(\pi^{-1}\right)^{\prime}(x)\right)=\pi \pi^{-1} \phi(x)
$$

and $\phi$ is an injection. Further if $x \leq y$ then $\phi(x) \leq \phi(y)$ and $\phi \pi^{\prime}(x)=\pi \phi(x) \leq \pi \phi(y)=\pi^{\prime}(y)$, so that $\pi^{\prime}(x) \leq \pi(y)$, since $\Omega^{\prime}$ is totally-ordered. We denote the map $\pi \rightarrow \pi^{\prime}$ from $A\left(\Omega^{\prime}\right)$ into $A(\Omega)$ by $A(\phi)$. Then calculations as above show that $A(\phi)$ is a lattice-group homomorphism (in general not injective, although $A(\phi)$ is, of course, an isomorphism onto $A(\Omega)$ when $A(\Omega)$ is transitive), and it is straightforward to check that this provides us with a (contravariant) functor from $A$ onto the category of ordered permutation groups of objects of $\underline{\underline{A}}$ and 
lattice-group homomorphisms.

COROLLARY 9. The map $\Omega \rightarrow \Phi(\Omega)$ is the object map of a contravariant functor from $\mathrm{A}$ into a category of distributive lattices.

Proof. We only have to check that if $\phi: \Omega^{\prime} \rightarrow \Omega$ is an arrow of $\underline{\underline{A}}$ and $\operatorname{fix}\left(\pi_{1}\right)=\operatorname{fix}\left(\pi_{2}\right)$ for $\pi_{1}, \pi_{2} \in A^{+}(\Omega)$ then $\operatorname{fix}\left(\pi_{1}^{\prime}\right)=\operatorname{fix}\left(\pi_{2}^{\prime}\right)$. If $\pi_{1}^{\prime}(x)=x$ then $\phi \pi_{1}^{\prime}(x)=\phi(x)$, so that $\pi_{1} \phi(x)=\phi(x)$, which gives $\pi_{2} \phi(x)=\phi(x)$ and so $\phi \pi_{2}^{\prime}(x)=\phi(x)$. Since $\phi$ is injective we have $\pi_{2}^{\prime}(x)=x$.

The category $\stackrel{A}{=}$ is somewhat unsatisfactory because of the terminal position in which it places those $\Omega$ with $A(\Omega)$ transitive and because all arrows in $\mathrm{A}$ are monic. It would be far better to obtain a category of totally-ordered sets for which $\Omega \rightarrow \Phi(\Omega)$ could be shown to be functorial without first going into a category of ordered permutation groups.

\section{Automorphic compactifications}

In this section we see that, under suitable but general restrictions, $K(\Omega)$ is an automorphic compactification of $\Omega$, and when $\Omega$ is excellent the Wallman compactification [5] of $\Omega$. is a Hausdorff automorphic compactification of $\Omega$.

THEOREM 10. If $\Omega$ is spectral and the subset

$$
T(\Omega)=\{\pi \in A(\Omega): \pi(x)>x \text { for all } x \in \Omega\}
$$

of $A(\Omega)$ is not empty then $K(\Omega)$ is an automorphic compactification of $\Omega$. If $\Omega$ is excellent then the Wallman compactification of $\Omega$ is $a$ Bcusdorff automorphic compactification.

Proof. Since $\Omega$ has no static points the support topology is defined. Further $\Phi(\Omega)$ has the empty subset $\square$ of $\Omega$ as a least element so $K(\Omega)$ is a compact $T_{0}^{\prime}$ space containing (a homeomorphic copy of) $\Omega$, since $\Omega$ is spectral. If $\xi \in K(\Omega)$ and $U(\pi)$ is a basic open neighbourhood of $\xi$ not meeting $\Omega$ then $f i x(\pi) \in \xi(x)$ for all $x \in \Omega$, so that $\operatorname{fix}(\pi)=\Omega$, and this contradicts $\operatorname{fix}(\pi) \neq \xi$. That is, $\Omega$ is dense in $K(\Omega)$. If $\pi \in A(\Omega)$ and $\xi \in K(\Omega)$ then we define 


$$
\bar{\pi}(\xi)=\left\{\pi \operatorname{fix}\left(\pi^{\prime}\right): \operatorname{fix}\left(\pi^{\prime}\right) \in \xi\right\}=\left\{\operatorname{fix}\left(\pi \pi^{\prime} \pi^{-1}\right): \operatorname{fix}\left(\pi^{\prime}\right) \in \xi\right\} .
$$

This provides us with a map from $K(\Omega)$ to $K(\Omega)$, since the elements of $A(\Omega)$ act as automorphisms of $\Phi(\Omega)$. Further, $\bar{\pi}$ is a bijection with inverse $\overline{\pi^{-1}}$. If $U\left(\pi_{1}\right)$ is a basic open set of $K(\Omega)$ then $\bar{\pi} U\left(\pi_{1}\right)=U\left(\pi \pi_{1} \pi^{-1}\right)$, so that $\bar{\pi}$ is an open map. The same thing applies to $\pi^{-1}$ so that $\bar{\pi}$ is a homeomorphism of $K(\Omega)$, which is an extension of $\pi$ since

$\bar{\pi}(\xi(x))=\left\{\operatorname{fix}\left(\pi \pi^{\prime} \pi^{-1}\right): \pi^{\prime}(x)=x\right\}=\left\{\operatorname{fix}\left(\pi^{\prime}\right): \pi^{\prime} \pi(x)=\pi(x)\right\}=\xi(\pi(x))$.

The Wallman compactification of $\Omega$ is just the set of maximal filters of $\Phi(\Omega)$ with the topology induced from $K(\Omega)$, and this is a Hausdorff space which is also an automorphic compactification of $\Omega$.

REMARK. The assumption that $T(\Omega) \neq \square$ ensures that $\square$ is the least element of $\Phi(\Omega)$ and so $K(\Omega)$ has a least element. It is not true that $\Phi(\Omega)$ always has the set of static points of $\Omega$ as a least element for if there were no static points then $\square$ would always be the least element of $\Phi(\Omega)$, and the following example, due to Ash, shows that this is not the case: let $\Omega$ be a totally ordered set obtained by replacing the first countable ordinal by the real interval $(0,1)$, and replacing the remaining countable ordinals by $[0,1)$. Then $\Omega$ has the following properties :

(a) $\Omega$ is Dedekind complete;

(b) every countable subset of $\Omega$ has a supremum in $\Omega$;

(c) $A(\Omega)$ is transitive;

(d) $\Omega$ has no static point's;

(e) $T(\Omega)=\square$.

\section{References}

[1] E. Bolz and G. Davis, "Compatible tight Riesz orders on ordered permutation groups", preprint. 
[2] Charles Holland, "Transitive lattice-ordered permutation groups", Math. Z. 87 (1965), 420-433.

[3] Justin T. Lloyd, "Complete distributivity in certain infinite permutation groups", Michigan Math. J. 14 (1967), 393-400.

[4] T.P. Speed, "Some topics in the theory of distributive lattices", (PhD thesis, Monash University, Clayton, Victoria, 1968).

[5] Henry Wallman, "Lattices and topological spaces", Arn. of Math. (2) 39 (1938), 112-126.

Department of Mathematics,

La Trobe University,

Bundoora, Victoria. 